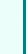

\title{
Superior Mesenteric Artery Syndrome
}

\author{
Efthimiou Matheos Kouritas Vasileios Baloyiannis loannis \\ Zacharoulis Dimitrios Hatzitheofilou Kostas
}

Department of Surgery, Larissa University Hospital, Mezourlo, Greece

\section{Key Words}

Superior mesenteric artery syndrome - Wilkie's syndrome - Duodenojejunostomy

\begin{abstract}
A 63-year-old female presented to our department complaining of epigastric pain, nausea and vomiting. Symptoms started after a significant loss of weight and persisted despite treatment, leading to hospitalization for dehydration and renal failure due to protracted vomiting. During hospitalization, no pathology could be identified and the patient was discharged. Symptoms persisted and she was eventually readmitted. Superior mesenteric artery syndrome was diagnosed based upon clinical suspicion and barium studies. She was subjected to duodenojejunostomy after failure of conservative treatment. Her immediate postoperative course was uneventful and the patient was well during her two-year follow-up. Clinicians should be suspicious of superior mesenteric artery syndrome, albeit rare, and be aware of its treatment, which is either conservative or surgical.
\end{abstract}

\section{Introduction}

Superior mesenteric artery syndrome (SMAS, also known as Wilkie's syndrome, aortomesenteric compression, arteriomesenteric duodenal compression or duodenal vascular compression) is a rare condition that results from vascular compression of the third part of the duodenum, in the angle between the aorta and the superior mesenteric artery. The syndrome is known since 1842; it was first observed by professor Rokitansky [1], but reported in the literature in 1927 by Wilkie [2]. Many articles were published thereafter including case reports and reviews [3]. Nevertheless, this syndrome still eludes most clinicians, and patients suffer from the disease for a long time before a firm diagnosis is reached. Therefore, any report illustrating the clinical or imaging appearance of this syndrome may be helpful in facilitating earlier diagnosis. We present the case of a 63 -year-old woman who was complaining of protracted vomiting for many months before being diagnosed with SMAS, possibly attributed to significant weight loss following cancer surgery. 


\begin{tabular}{|c|c|c|}
\hline asmo-nterelay & DOI: $10.1159 / 000209866$ & $\begin{array}{l}\text { ISSN 1662-0631 } \\
\text { www.karger.com/crg }\end{array}$ \\
\hline
\end{tabular}

\section{Case Presentation}

A 63-year-old female was admitted to our department complaining of anorexia, vomiting, dyspepsia and intermittent epigastric abdominal pain. Her past medical history included a total hysterectomy for cervical cancer five years earlier. Her presenting complaints initially appeared one month following a significant and rapid loss of weight (10 kg in one month) a year before. She was treated at the time with proton pump inhibitors but her symptoms worsened, with vomiting being added to her complaints. A few months later she was hospitalized at a tertiary hospital due to protracted vomiting and dehydration which led to renal failure. During that hospitalization she was subjected to gastroscopy, colonoscopy and abdominal CT scan which did not reveal any pathologies. Gastritis was the final diagnosis and the patient was discharged. However, her symptoms persisted and she was finally admitted to our department. Gastroscopy and colonoscopy were repeated and were reported to be normal, whereas the barium meal revealed dilatation of the proximal duodenum, stenosis of its third part and late progression of barium to the jejunum (ig. 1). Based on the history of the complaints, the patient's clinical appearance and the barium meal, SMAS was suspected.

A nasogastric tube was inserted and the patient was started on parenteral nutrition. Conservative measures failed, and 2 weeks later the patient was subjected to laparotomy, where dilatation of the duodenum proximally to the superior mesenteric vessels was observed. Injection of $200 \mathrm{ml}$ of normal saline [4] through the nasogastric tube resulted in further dilatation of the proximal duodenum. The third part of the duodenum was exposed and mobilized and a loop duodenojejunostomy was performed, approximately $10 \mathrm{~cm}$ distally to the ligament of Treitz (fig. 2$)$. Her postoperative course was uneventful. A barium meal repeated 30 days postoperatively showed a normal duodenum with uninterrupted passage of barium to the jejunum (ig. 3 ).

\section{Discussion}

SMAS is a rare condition which is due to anatomical or mechanical abnormalities. Clinicians need a high degree of suspicion in order to diagnose SMAS. The reported prevalence of this syndrome in the general population varies between 0.013 and $0.3 \%$ [5], although by applying stricter clinical or imaging criteria this rate may be reduced even further [6].

Numerous predisposing factors for SMAS have been reported and can be roughly categorized into three major groups [3]: (1) severe weight loss (i.e. in chronic debilitating disease, dietary disorders and malabsorption), (2) external causes (i.e. scoliosis surgery with instrumentation or body casting, ileoanal pouch anastomosis), and (3) intraabdominal compression or mesenteric tension (i.e. neoplastic growth, aortic aneurysm). In our case, weight loss following pelvic surgery for cervical cancer was the probable predisposing factor that led to obstruction of the duodenum, which was surgically verified, with no other causes of obstruction identified intraoperatively.

The precursor symptom of the syndrome is postprandial abdominal pain with associated nausea, vomiting and anorexia [5]. The pain is characteristically relieved by prone, knee-chest or left lateral decubitus position [5]. Patients may complain of chronic abdominal pain, the severity of which depends on the cause of obstruction and may run for months or even years. In rare conditions the syndrome may present acutely, mimicking upper intestinal ileus [7]. In our case, the disease had gradually exacerbated after a period of weight loss following cancer surgery. The clinical appearance was acute as the patient ended up with extensive electrolyte imbalances which led to renal failure.

Diagnosis is established by all kinds of imaging techniques, including computed tomography (CT), CT angiography, ultrasonography and magnetic resonance imaging (MRI) [8-10]. Recently, even more complex imaging studies have been developed, using multidetector CT angiography and three-dimensional reconstruction [10]. CT and contrast-enhanced CT as imaging modalities allow direct visualization of the obstructed 
bowel in relation to vessels, such as the superior mesenteric artery [10]. Additionally it can demonstrate the aortomesenteric angle, its distance from the bowel and the amount of retroperitoneal fat tissue, as well as other possible causes of obstruction that may warrant urgent surgical treatment, such as aneurysm [3]. Nonetheless, barium studies are still important for the diagnosis of SMAS. A classic picture obtained is a dilated proximal duodenum with an abrupt termination of the barium column in the third part [3]. However, this appearance may be seen in other pathologies, such as in megaduodenum [7]. Therefore, more stringent criteria have been established for the diagnosis of SMAS, including (1) dilatation of the first and second parts of the duodenum, (2) abrupt vertical and oblique compression of the mucosal folds, (3) antiperistaltic flow of the contrast medium proximal to the obstruction, (4) delay in transit of 4-6 $\mathrm{h}$ through the gastroduodenal region, and (5) relief of the obstruction in a prone, knee-chest or left lateral decubitus position [6]. Finally, endoscopy should be performed to exclude intestinal lumen obstruction or ulcer disease mimicking the syndrome. The facilities of contrast CT or MRI are not available in our hospital and therefore the preoperative diagnosis was based solely on barium study.

Treatment is initially conservative, including the insertion of a nasogastric tube, mobilization of the patient to a prone, left lateral decubitus position, administration of parenteral nutrition, fluid-electrolyte balance correction and positive nitrogen balance to increase body weight and in this way restore the retroperitoneal fat tissue [3]. In our patient, conservative treatment failed, and surgery was undertaken in order to avoid the risk of duodenal atony and massive dilatation. There is no clear time point to operate, but if the patient remains symptomatic after 2-12 days of conservative treatment, the surgical option should be considered [4].

Surgical treatment of SMAS involves a wide range of procedures, including Treitz ligament division (Strong's operation), gastrojejunostomy, subtotal gastrectomy and Billroth II gastrojejunostomy, duodenojejunostomy, anterior reposition of the duodenum, duodenal derotation [11] and duodenal circular drainage operation [12]. Superior mesenteric artery transposition surgery has also been described [13]. Duodenojejunostomy was initially performed in 1908 and was the first operation conducted for the treatment of SMAS. For many years it was considered the best surgical option $[3,4,14]$. Although duodenojejunostomy is still the most frequently conducted procedure for SMAS, it has recently been reported that it does not alleviate vomiting [12], possibly due to the reversed peristalsis which in some cases is greater than the direct peristalsis or because of gastroparesis following correction surgery. Yang and Zhang [12] proposed the duodenal circular drainage operation to overcome this drawback usually observed in duodenojejunostomy, however this type of procedure has some disadvantages. Finally, laparoscopic techniques have made SMAS correction surgery feasible, but the number of patients included in these studies is relatively small $[3,14,15]$.

In conclusion, clinicians should always be aware of SMAS despite its low incidence. Nowadays, CT or MRI scans can establish the diagnosis; nonetheless, classic barium study with application of strict criteria may still be of value. Duodenojejunostomy is the most frequent surgical procedure performed after conservative treatment failure, but recent data suggest that it may not relieve symptoms entirely. SMAS is a rare entity and unfortunately recent, large-scale studies do not exist so far. 


\begin{tabular}{r|l|l|l} 
Case Reports $/ \mathrm{n}$ & $\begin{array}{l}\text { Case Rep Gastroenterol 2009;3:156-161 } \\
\text { D01: 10.1159/000209866 }\end{array}$ & Published online: May 27, 2009 & $\begin{array}{l}\text { 2009 S. Karger AG, Basel } \\
\text { ISSN 1662-0631 } \\
\text { Www.karger.com/crg }\end{array}$ \\
\hline
\end{tabular}

Fig. 1. Preoperative barium study. Note dilatation of the first and second parts of the duodenum and delay in transit of $4-6 \mathrm{~h}$ through the gastroduodenal region.

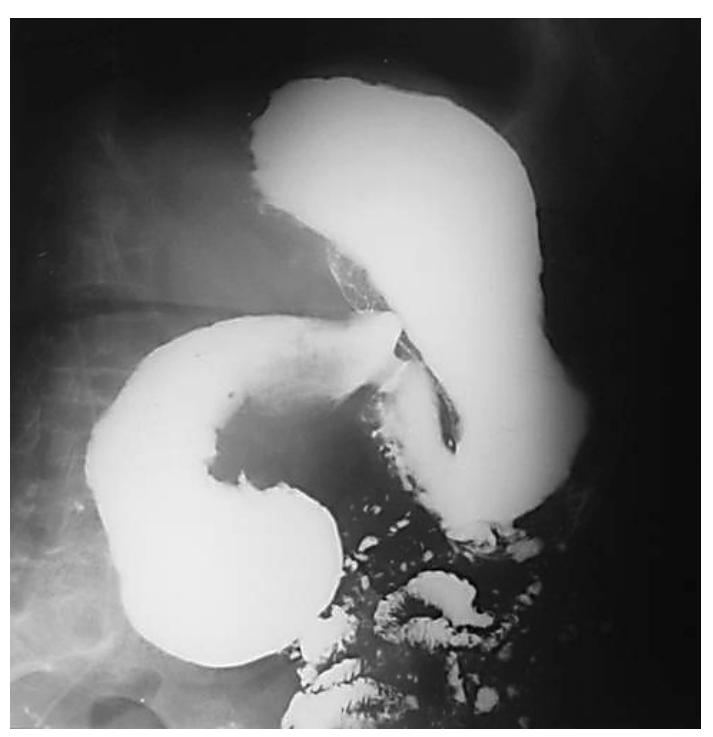

Fig. 2. Duodenojejunostomy.

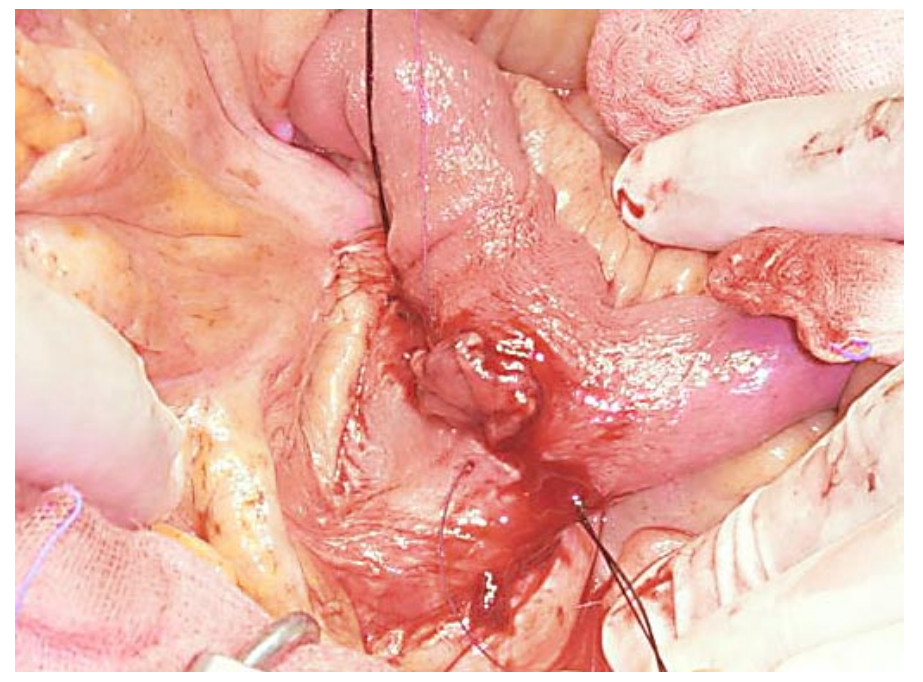


Case Reports in | Case Rep Gastroenterol 2009;3:156-161 Case Rep Gastroenterol 2009
D0I: $10.1159 / 000209866$

Fig. 3. Postoperative barium study.

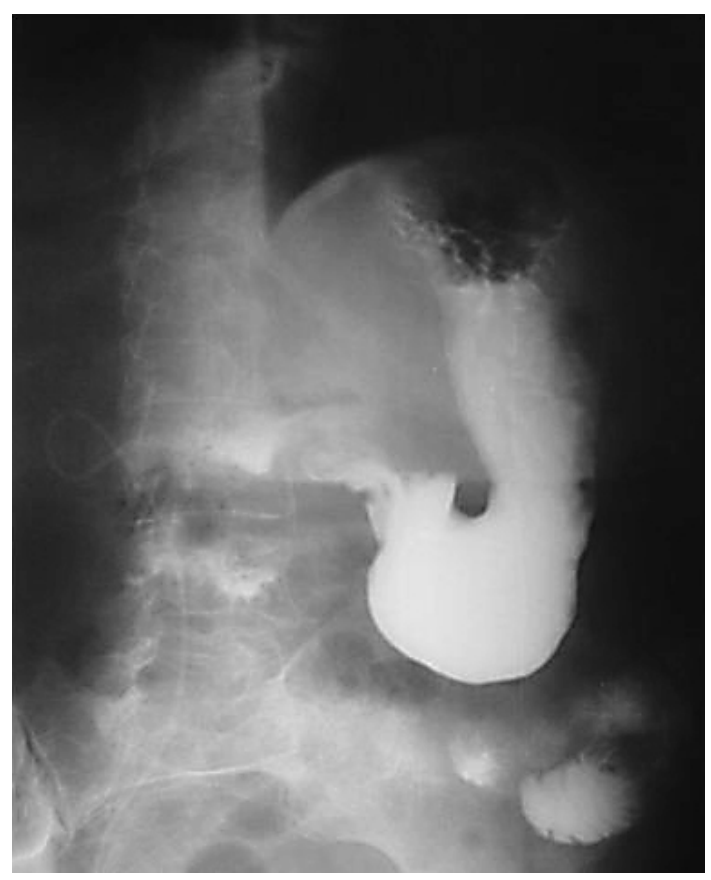




\section{References}

1 Rokitansky C: Handbuch der pathologischen Anatomie, ed 1. Wien, Braunmüller \& Seidel, 1842, vol 3, p 187.

2 Wilkie DPD: Chronic duodenal ileus. Am J Med Sci 1927;173:643-649.

-3 Welsch T, Buchler MW, Kienle P: Recalling superior mesenteric artery syndrome. Dig Surg 2007;24:149-156.

4 Cheshire NJ, Glezer G: Diverticula, volvuluvs, superior mesenteric artery syndrome, and foreign bodies; in Zinner MJ, Schwartz SI, Ellis H (eds): Maingot's Abdominal Operations, vol I, ed 9. East Norwalk, Conn., Appleton \& Lange, 1990, pp 587-591.

5 Ylinen P, Kinnunen J, Hockerstedt K: Superior mesenteric artery syndrome. A follow-up study of 16 operated patients. J Clin Gastroenterol 1989;11:386-391.

-6 Hines JR, Gore RM, Ballantyne GH: Superior mesenteric artery syndrome. Diagnostic criteria and therapeutic approaches. Am J Surg 1984;148:630-632.

7 Kwan E, Lau H, Lee F: Wilkie's syndrome. Surgery 2004;135:225-227.

8 Unal B, Aktas A, Kemal G, Bilgili Y, Guliter S, Daphan C, Aydinuraz K: Superior mesenteric artery syndrome: CT and ultrasonography findings. Diagn Interv Radiol 2005;11:90-95.

-9 Konen E, Amitai M, Apter S, Garniek A, Gayer G, Nass S, Itzchak Y: CT angiography of superior mesenteric artery syndrome. AJR Am J Roentgenol 1998;171:1279-1281.

10 Agrawal GA, Johnson PT, Fishman EK: Multidetector row CT of superior mesenteric artery syndrome. J Clin Gastroenterol 2007;41:62-65.

11 Ha CD, Alvear DT, Leber DC: Duodenal derotation as an effective treatment of superior mesenteric artery syndrome: a thirty-three year experience. Am Surg 2008;74:644-653.

12 Yang W, Zhang X: Assessment of duodenal circular drainage in treatment of superior mesenteric artery syndrome. World J Gastroenterol 2008;14:303-306.

13 Pourhassan S, Grotemeyer D, Fürst G, Rudolph J, Sandmann W: Infrarenal transposition of the superior mesenteric artery: a new approach in the surgical therapy for Wilkie syndrome. J Vasc Surg 2008;47:201-204.

14 Palanivelu C, Rangarajan M, Senthilkumar R, Parthasarathi R, Jani K: Laparoscopic duodenojejunostomy for superior mesenteric artery syndrome. JSLS 2006; 10:531-534.

15 Jo JB, Song KY, Park CH: Laparoscopic duodenojejunostomy for superior mesenteric artery syndrome: report of a case. Surg Laparosc Endosc Percutan Tech 2008;18:213-215. 